\title{
Individually selected teletherapy technique for accelerated partial breast irradiation
}

\author{
Renáta L. Kószó ${ }^{1}$, Zsuzsanna Kahán ${ }^{1}$, Barbara Darázs ${ }^{1}$, Ferenc Rárosi ${ }^{2}$, Zoltán Varga ${ }^{1}$ \\ ${ }^{1}$ Department of Oncotherapy, Faculty of Medicine, University of Szeged, Szeged, Hungary \\ ${ }^{2}$ Department of Medical Informatics, Faculty of Medicine, Faculty of Science and Informatics, University of Szeged, Szeged, Hungary
}

\begin{abstract}
Background: The aim of the study was to individualize accelerated partial breast irradiation based on optimal dose distribution, protect risk organ and predict most advantageous technique.

Materials and methods: 138 breast cancer patients receiving postoperative APBI were enrolled. APBI plans were generated using 3D-conformal (3D-CRT), sliding window intensity-modulated radiotherapy (IMRT) and volumetric-modulated arc therapy (VMAT). In the case of superficial tumours, additional plans were developed by adding electron beam. To planning target volume (PTV) 37.5 Gy/10 fractions, 1 fraction/day was prescribed. A novel plan quality index (PQI) served as the basis for comparisons.

Results: IMRT was the most advantageous technique regarding homogeneity. VMAT provided best conformity, 3D-CRT — the lowest lung and heart exposure. PQI was the best in 45 (32.61\%) VMAT, 13 (9.42\%) IMRT, 9 (6.52\%) 3D-CRT plans. In 71 cases (51.45\%) no difference was detected. In patients with large PTV, 3D-CRT was the most favourable. Additional electron beam improved PQI of 3D-CRT plans but had no meaningful effect on IMRT or VMAT. IMRT was superior to VMAT if the tumour was superficial $(p<0.001)$, situated in the medial $(p=0.032)$ or upper quadrant $(p=0.046)$.

Conclusions: In half of all cases, individually selected teletherapy techniques provide superior results over others; relevance of a certain technique may be predicted by volume and PTV localization.
\end{abstract}

Key words: accelerated partial breast irradiation; conformal radiotherapy; intensity-modulated radiotherapy; plan quality index; volumetric-modulated arc therapy

Rep Pract Oncol Radiother 2021;26(6):990-1002

\section{Introduction}

Breast cancer is the most frequent female malignancy, but due to mammography screening, about $40 \%$ of cancers are diagnosed at a truly early stage and many times managed with breast conserving surgery and radiation therapy $[1,2]$. Accelerated partial breast irradiation (APBI) introduced into practice about 10 years ago, is an appropriate treatment method in selected low-risk patients [2-5].
Nowadays, based on the efficacy and safety results, APBI may also be considered in cases formerly categorized as medium-risk [7].

A traditional approach for the implementation of APBI has been brachytherapy, with the use of needles or balloon catheter $[2,3,6]$. Due to the rising number of patients needing APBI, and to eliminate the special infrastructural and expertise needs of brachytherapy, the implementation of conformal teletherapy methods, such as $3 \mathrm{D}$-conformal

Address for correspondence: Renáta L. Kószó, Department of Oncotherapy, University of Szeged, Korányi alley 12, H-6720 Szeged, Hungary, tel: +36 62545 404, fax: +36 62545 922; e-mail: koszo.renata.lilla@med.u-szeged.hu 
radiation therapy (3D-CRT) with multiple static photon and/or electron fields, intensity-modulated RT (IMRT), volumetric-modulated arc therapy (VMAT), tomotherapy, robotic radiosurgery systems and proton beam therapy has begun emerging [8-11]. The utilization of a mixed technique combining photon with an 'en face' electron field may result in improved planning target volume (PTV) coverage and organ at risk (OAR) exposure [10-14]. IMRT optimizes dose distribution using structure-based planning and alterable intensity beam fluencies, providing improved dose homogeneity within the PTV, and reduced high dose exposure of normal tissues. However, applying multiple beams increases the healthy tissue volume exposed to low and moderate doses. Dose homogeneity and OAR exposure may be further improved by gantry rotation combined with dynamic multileaf collimation specific for VMAT $[8,9]$. At the level of individuals, there may be differences in the RT plan quality among the techniques. There are several factors reflecting conformity, homogeneity, PTV-coverage, and OAR exposure [15-17]; however, all these characteristics describe one certain aspect of a plan only.

Our goal was to perform personalized APBI regarding dose distribution and healthy tissue protection and identification of tumour- and patient-related characteristics for selecting the optimal irradiation method (3D-CRT, IMRT, VMAT or photon-electron mixed beam RT). For the complex comparison of RT plans and appropriate APBI plan selection for each patient, the Plan Quality Index (PQI) method was adapted, originally implemented for prostate IMRT plan evaluation [18].

\section{Materials and methods}

\section{Patient population}

Women after breast conserving surgery, with at an age of at least 50 years were enrolled into this dosimetry study. Inclusion criteria were a unifocal and unicentric tumour of any invasive histological type or low-risk ductal carcinoma in situ (DCIS) of any hormone receptor and human epidermal growth factor receptor-2 (HER2) status, pT1-2 $(\leq 30 \mathrm{~mm})$ tumour size, a resection margin of $\geq 2 \mathrm{~mm}, \mathrm{pN} 0$ axillary status determined by sentinel lymph node biopsy or axillary block dissection, the lack of extensive intraductal component (EIC) and lympho- vascular invasion, an M0 status, and the presence of titanium clips indicating the tumour bed. The existence of any relative and absolute contraindication of RT represented the exclusion criteria. Clinical data including tumour bed location (upper, lower, lateral, medial/central quadrant) were collected.

\section{Patient positioning and planning CT scanning}

Patients were positioned in a supine posture with arms raised above the head using an "All in One (AIO) Solution" (ORFIT, Wijnegem, Belgium) breast board device. Immobilization was carried out with diagonal thermoplastic masks (ORFIT, Wijnegem, Belgium). In every case, five-millimetre slice-increment topometric computed tomography (CT) scans were taken from the sternoclavicular joint to $2 \mathrm{~cm}$ below the submammary fold, using a Somatom Emotion 6 CT Simulator (Siemens, Erlangen, Germany).

\section{Target and risk organ contouring}

The clinical target volume (CTV) encompassed the surgical cavity (localized with titanium clips) with a $15 \mathrm{~mm}$ extension in all directions. The ventral and dorsal border was cropped $4 \mathrm{~mm}$ under the skin and by the chest wall, respectively. Planning target volume (PTV) was generated by adding a 5 $\mathrm{mm}$ margin to the CTV for compensating setup errors and breathing motions. The lung, heart, left descending coronary artery (LAD) [19,20], ipsilateral and contralateral breast were contoured as OARs.

\section{Treatment planning}

3D-CRT, sliding window IMRT and VMAT plans were created for a Varian TrueBeamSTx (Varian Oncology Systems, Palo Alto, CA, USA) linear accelerator equipped with HD120 multileaf collimator, using the Eclipse v13.6 planning system (Varian Oncology Systems, Palo Alto, CA, USA) in each case. In 3D-CRT plans, two $6 \mathrm{MV}$ photon fields were employed, angled at approximately $120^{\circ}$ (Fig. 1A). Field direction was based on the tumour bed situation and in left-sided cases, on the position of the heart and LAD. Additional sub-segments were administered to improve homogeneity, if necessary. Sliding window IMRT plans were generated employing five $6 \mathrm{MV}$ photon fields with a beam arrangement of $300^{\circ}, 350^{\circ}, 40^{\circ}, 90^{\circ}, 150^{\circ}$ in left-sided cases and $60^{\circ}, 10^{\circ}, 320^{\circ}, 270^{\circ}, 210^{\circ}$ in 

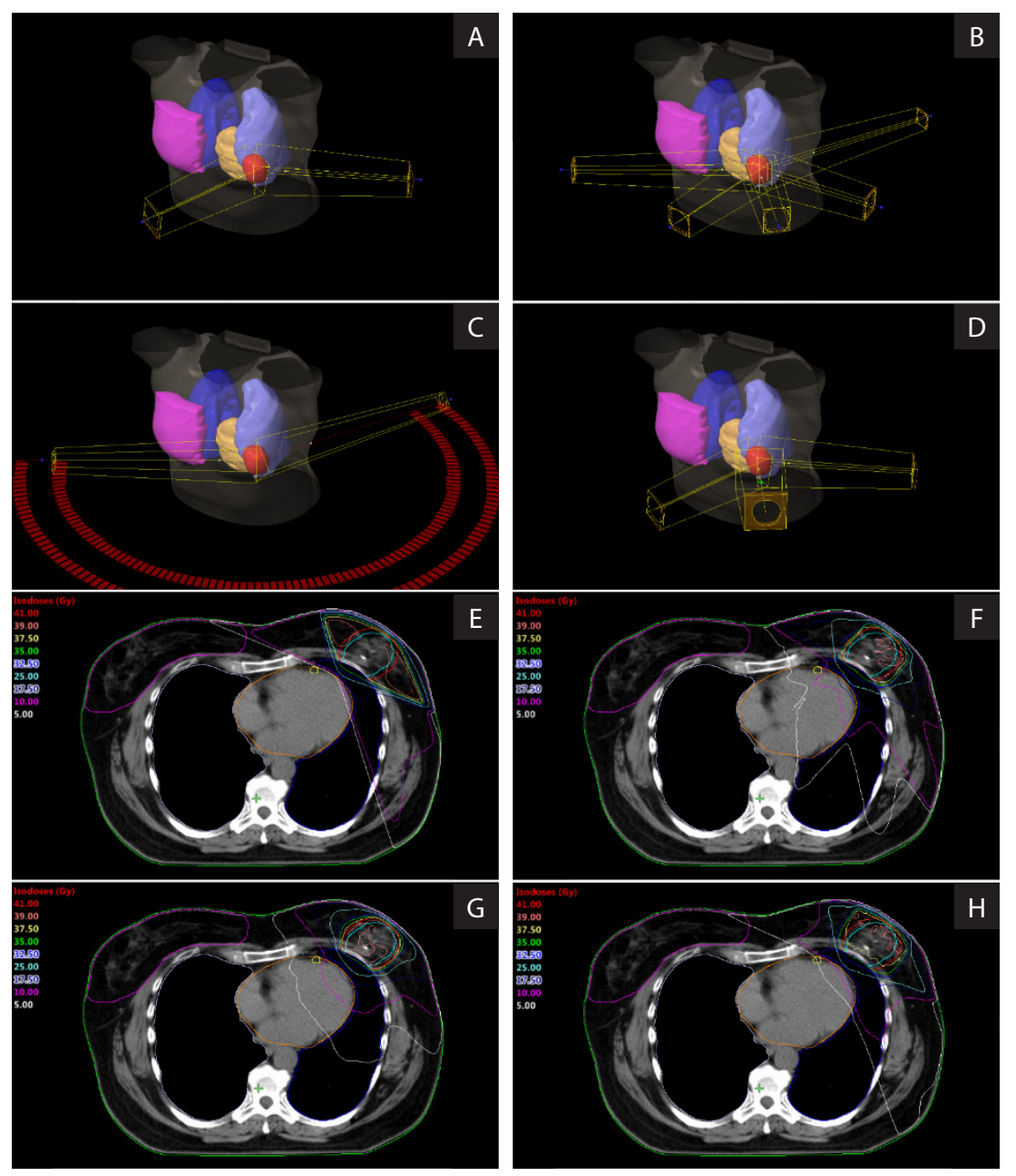

Figure 1. Beam arrangements and dose distributions. 3 dimensional conformal radiation therapy (3D-CRT) (A), intensity modulated radiation therapy (IMRT) (B), volumetric modulated arc therapy (VMAT) (C) radiotherapy techniques and the combination of photon fields with an "en face" electron beam (D); dose distribution in the various plans are shown: 3D-CRT $(\mathbf{E}), \operatorname{IMRT}(\mathbf{F}), \operatorname{VMAT}(\mathbf{G})$, photon fields with an "en face" electron beam $(\mathbf{H})$

right-sided cases (Fig. 1B). Dual arc VMAT field arrangement was determined by the outermost IMRT fields (Fig. 1C). The isocentre was placed in the geometric centre of the target volume. Inverse plans were optimized using the same parameters for comparability purposes. A 4-16 MeV energy 'en face' electron beam was added to each technique if the distance between the geometric centre of the target volume and the skin surface (d) was $<25 \mathrm{~mm}$ (Fig. 1D). Two-thirds of the dose was reckoned with photons and one-third with electrons. For shaping the electron beams, Newton's metal apertures were used. Dose distribution in the various plans is illustrated in Figures 1E-H. Ten fractions of fraction doses of 3.75 Gy were prescribed for the PTV using daily fractionation, 5 treatments/week. Plan normalization was carried out according to the requirement that $99 \%$ of the PTV receives $95 \%$ of the prescribed dose. $\geq 90 \%$ of the PTV was to be covered with $100 \%$ of the prescribed dose, and $\leq 10 \%$ of the PTV was to be exposed to $>107 \%$ of the prescribed dose.

\section{Plan evaluation}

For each plan, conformity and homogeneity indices were calculated and some dose-volume parameters of the OARs were collected. The conformation number $(\mathrm{CN})$ [15], homogeneity index (HI) [16] were calculated as described.

$$
\begin{gathered}
C N=\frac{P T V_{\text {ref }}}{V_{P T V}} \times \frac{P T V_{\text {ref }}}{V_{\text {ref }}} \quad(\text { Ideal is } 1) \\
H I=\frac{D_{2 \%}-D_{98 \%}}{D_{50 \%}}(\text { Ideal is } 0)
\end{gathered}
$$


Plan quality index (PQI) developed by Leung et al. [18] was adapted to APBI to characterize treatment plans with a single number.

$$
P Q I=\sqrt{(1-H)^{2}+(1-M)^{2}+(1-P)^{2}}(\text { Ideal is } 0)
$$

The $(\mathrm{H})$ ealthy tissue conformity index [17]:

$$
H=\frac{P T V_{\text {ref }}}{V_{\text {ref }}}(\text { Ideal is } 1)
$$

PTV coverage was described with the "(M)erit function" parameter [18], representing hot and cold spots within the PTV. The percentage PTV volume covered with the $100 \%$ isodose curve (min. 90\%) was determined as cold spots, the percentage PTV volume exposed to at least $107 \%$ of the prescribed dose (max. 10\%) was defined as hot spots [18].

Data regarding the relative ipsilateral breast volume (ipsilateral breast - PTV) covered with min. 25 or $75 \%$ of the prescribed dose $\left(\right.$ Breast $\mathrm{V}_{25 \%}$ and Breast $\mathrm{V}_{75 \%}$, respectively), mean ipsilateral lung exposure (Lung mean ) and relative ipsilateral lung volume receiving $\geq 40 \%$ of the prescribed dose $\left(\right.$ Lung $\left.\mathrm{V}_{40 \%}\right)$, mean heart dose $\left(\mathrm{Heart}_{\text {mean }}\right)$ and relative heart volume receiving $\geq 50 \%$ of the prescribed dose $\left(\right.$ HeartV $\left.\mathrm{V}_{50 \%}\right)$, average LAD dose $\left(\mathrm{LAD}_{\text {mean }}\right)$ and relative LAD volume covered with at least $20 \%$ of the prescribed dose $\left(\mathrm{LADV}_{20 \%}\right)$ were registered.

For characterizing OAR exposure during APBI, the formula of Leung et al. [18] was modified as follows. Selected dose parameters of four risk organs related to the $99 \%$ percentile of the given sample population were averaged to create the ' $(\mathrm{P})$ enalty function' parameter [18] specific for a given RT technique.

In right-sided cases:

$$
\left.P=\frac{\left(1-\frac{\text { BreastV }_{25 \%}}{70}\right)+\left(1-\frac{\text { Lung }_{\text {mean }}}{10}\right)+\left(1-\frac{\text { Heart }_{\text {mean }}}{5}\right)+\left(1-\frac{L A D_{\text {mean }}}{5}\right)}{4} \text { (Ideal is } 1\right)
$$

In left-sided cases:

$P=\frac{\left(1-\frac{\text { Breast }_{25 \%}}{70}\right)+\left(1-\frac{\text { Lung }_{\text {mean }}}{10}\right)+\left(1-\frac{\text { Heart }_{\text {mean }}}{10}\right)+\left(1-\frac{L A D_{\text {mean }}}{10}\right)}{4}$ (Ideal is 1$)$

A negative $\mathrm{P}$ value (in case of high dose to all OARs) is to be calculated as 0 .

For prioritizing RT plans in individual cases, the PQI values were compared. To determine an arbitrary threshold of PQI difference that indicates a difference in about half of the cases, we defined the PQI difference (PQID) as relevant if it exceeded the value of 0.05 . Each plan that reached this critical PQID level was referred to a respective 'winner method group', while that which did not was referred to the group of equality. To identify the RT method optimal for a certain subgroup of patients, the effects of the PTV volume, the distance between the target volume and the skin surface (d) and the breast quadrant where the tumour bed was located were studied.

\section{Ethical approval}

The study had been approved by the Regional Human Biomedical Research Ethics Committee, University of Szeged, Hungary (74/2015-SZTE). All patients gave their written informed consent before entering the study.

\section{Statistical methods}

Continuous variables were represented as mean \pm standard deviation (SD). The means of continuous variables in the 'winner method groups' were compared with Welch's one-way ANOVA. Multiple comparisons were performed using the least significant difference (LSD) method after significant ANOVA. Dependency between two categorical variables was studied with Pearson's Chi-squared tests. Pearson correlation coefficients were calculated. Impact of an added electron beam to photon beams on PQI (3D-CRT vs. IMRT vs. VMAT) was studied using two-way repeated measures (within subjects-within subjects) ANOVA method. A p $<0.05$ was considered as statistically significant. For statistical analysis, the IBM SPSS version 24 software was used.

\section{Results}

\section{Patient population}

138 patients were enrolled into the study in 2 cohorts ( $\mathrm{n}=72$, November 2009 - December 2012 and $\mathrm{n}=66$, September 2015 - July 2018). The first cohort received 3D-CRT (photon or electron) [10], the other cohort VMAT $(\mathrm{n}=58)$, IMRT $(\mathrm{n}=2)$, 3D-CRT $(\mathrm{n}=2)$ or photon-electron mixed technique $(\mathrm{n}=4)$. The actual radiotherapy technique was selected according to availability. The median age was 62.0 (50.1-79.7) years, and most of the patients were postmenopausal. Most cancers were detected via screening, and in the outer-upper breast quadrant. Histology was mostly invasive 
ductal carcinoma of grade 1-2, hormone receptor positive and HER2-negative. The mean \pm SD of the pathologic tumour extension was $11.3 \pm 4.7$ $\mathrm{mm}$, the average $\pm \mathrm{SD}$ surgical resection margin was $6.8 \pm 4.1 \mathrm{~mm}$.

\section{Radiotherapy data}

The target volume was in the left breast in 78 patients $(56.5 \%)$ and in the right breast in 60 patients (43.5\%). The average and median PTV volumes were $115.6 \mathrm{~cm}^{3}$ and $108.5(23.7-287.8) \mathrm{cm}^{3}$, respectively. The PTV volume was at least $100 \mathrm{~cm}^{3}$ in 75 patients $(54.3 \%)$. The mean \pm SD distance between the centre of the PTV and the skin surface (d) was $3.6 \pm 1.6 \mathrm{~cm}$, while in 29 cases $(21.0 \%)$ it was $<25 \mathrm{~mm}$.

In most cases, the IMRT and VMAT techniques showed superior PQI values. According to the dose homogeneity and conformity data, in most cases homogeneity was improved by IMRT, and the best conformity was provided by VMAT (Tab. 1). OAR doses were diverse, but the $3 \mathrm{D}$-CRT technique provided the lowest mean heart and lung doses (Tab. 2). These results point to the fact that traditional plan quality indicators per se are not suitable to choose the optimal technique in an individual case (Tab. 1, 2). "H", "M" and "P" parameters and
PQI values are summarized in Table 3. Analysis of 3D-CRT, IMRT and VMAT techniques based on the PQID $>0.05$ threshold in the whole cohort demonstrated that the three methods were equally suitable in 71 cases (51.45\%), VMAT was the most favourable technique in 45 cases (32.61\%), IMRT was optimal in 13 patients $(9.42 \%)$ and 3D-CRT was the best in 9 cases $(6.52 \%)$.

Comparison of the two inverse treatment planning techniques separately using the PQID $\geq 0.05$ revealed that VMAT plans were the best in 55 cases (39.9\%) and IMRT plans were preferable in 14 cases $(10.1 \%)$, while both were equally good in 69 patients (50.0\%).

Analysing the PQI indicators of cases with 3DCRT as the optimal technique to those for whom 3D-CRT was either equally good or worse than IMRT and VMAT, only the PTV volume proved to be a significant variable ( $\mathrm{p}=0.017$ ) (Fig. 2). The average \pm SD PTV volume was $159.3 \pm 67.9 \mathrm{~cm}^{3}$ in patients for whom the best technique was $3 \mathrm{D}$ CRT, $114.4 \pm 46.3 \mathrm{~cm}^{3}$ in those for whom IMRT performed the best, $102.9 \pm 50.9 \mathrm{~cm}^{3}$ in those for whom VMAT was the most favourable technique, and $118.3 \pm 44.8 \mathrm{~cm}^{3}$ in those for whom all three methods resulted similar PQI values. Post hoc tests showed that the volume of the PTV was larger if

Table 1. Partial breast irradiation according to the radiotherapy technique used: parameters reflecting dose distribution within the planning target volume (PTV) and conformity

\begin{tabular}{|c|c|c|c|c|c|}
\hline & Technique & $\begin{array}{c}\mathrm{V} 100 \% \\
(\text { mean } \pm \mathrm{SD}, \%)\end{array}$ & $\begin{array}{c}\mathrm{V} 107 \% \\
(\text { mean } \pm \mathrm{SD}, \%)\end{array}$ & $\begin{array}{c}\mathrm{CN} \\
(\text { mean } \pm \mathrm{SD})\end{array}$ & $\begin{array}{c}\mathrm{HI} \\
(\text { mean } \pm \mathrm{SD})\end{array}$ \\
\hline \multirow{3}{*}{ All cases } & 3D-CRT & $96.27 \pm 1.46$ & $3.51 \pm 1.53$ & $0.582 \pm 0.063$ & $0.083 \pm 0.018$ \\
\hline & IMRT & $96.16 \pm 1.64$ & $0.68 \pm 0.73$ & $0.833 \pm 0.081$ & $0.045 \pm 0.010$ \\
\hline & VMAT & $96.71 \pm 0.87$ & $1.45 \pm 1.16$ & $0.901 \pm 0.032$ & $0.054 \pm 0.010$ \\
\hline \multirow{3}{*}{ PTV $<100 \mathrm{~cm}^{3}$} & 3D-CRT & $96.30 \pm 1.36$ & $3.46 \pm 1.51$ & $0.585 \pm 0.061$ & $0.082 \pm 0.018$ \\
\hline & IMRT & $95.85 \pm 2.27$ & $0.66 \pm 0.79$ & $0.808 \pm 0.090$ & $0.046 \pm 0.011$ \\
\hline & VMAT & $96.54 \pm 1.16$ & $1.50 \pm 1.33$ & $0.900 \pm 0.035$ & $0.054 \pm 0.011$ \\
\hline \multirow{3}{*}{ PTV $\geq 100 \mathrm{~cm}^{3}$} & 3D-CRT & $96.26 \pm 1.55$ & $3.56 \pm 1.55$ & $0.580 \pm 0.065$ & $0.085 \pm 0.017$ \\
\hline & IMRT & $96.42 \pm 0.72$ & $0.69 \pm 0.67$ & $0.853 \pm 0.066$ & $0.044 \pm 0.010$ \\
\hline & VMAT & $96.86 \pm 0.46$ & $1.40 \pm 1.00$ & $0.902 \pm 0.030$ & $0.055 \pm 0.009$ \\
\hline \multirow{6}{*}{$\mathrm{d}<2.5 \mathrm{~cm}$} & 3D-CRT & $96.56 \pm 0.75$ & $3.86 \pm 1.29$ & $0.589 \pm 0.068$ & $0.089 \pm 0.016$ \\
\hline & 3D-CRT+e & $94.75 \pm 2.35$ & $4.71 \pm 1.55$ & $0.765 \pm 0.071$ & $0.082 \pm 0.014$ \\
\hline & IMRT & $95.85 \pm 3.20$ & $1.07 \pm 0.91$ & $0.785 \pm 0.081$ & $0.052 \pm 0.010$ \\
\hline & IMRT+e & $94.20 \pm 3.42$ & $2.87 \pm 1.39$ & $0.828 \pm 0.069$ & $0.060 \pm 0.008$ \\
\hline & VMAT & $96.52 \pm 1.65$ & $2.35 \pm 1.41$ & $0.870 \pm 0.037$ & $0.064 \pm 0.007$ \\
\hline & VMAT+e & $95.75 \pm 2.19$ & $3.26 \pm 1.34$ & $0.886 \pm 0.048$ & $0.065 \pm 0.008$ \\
\hline
\end{tabular}

IMRT — intensity modulated radiation therapy; VMAT — volumetric modulated arc therapy; 3D-CRT — 3 dimensional conformal radiation therapy; $\mathrm{SD}$ - standard deviation; $\mathrm{Cl}$ - conformation number; $\mathrm{HI}$ - homogeneity index 


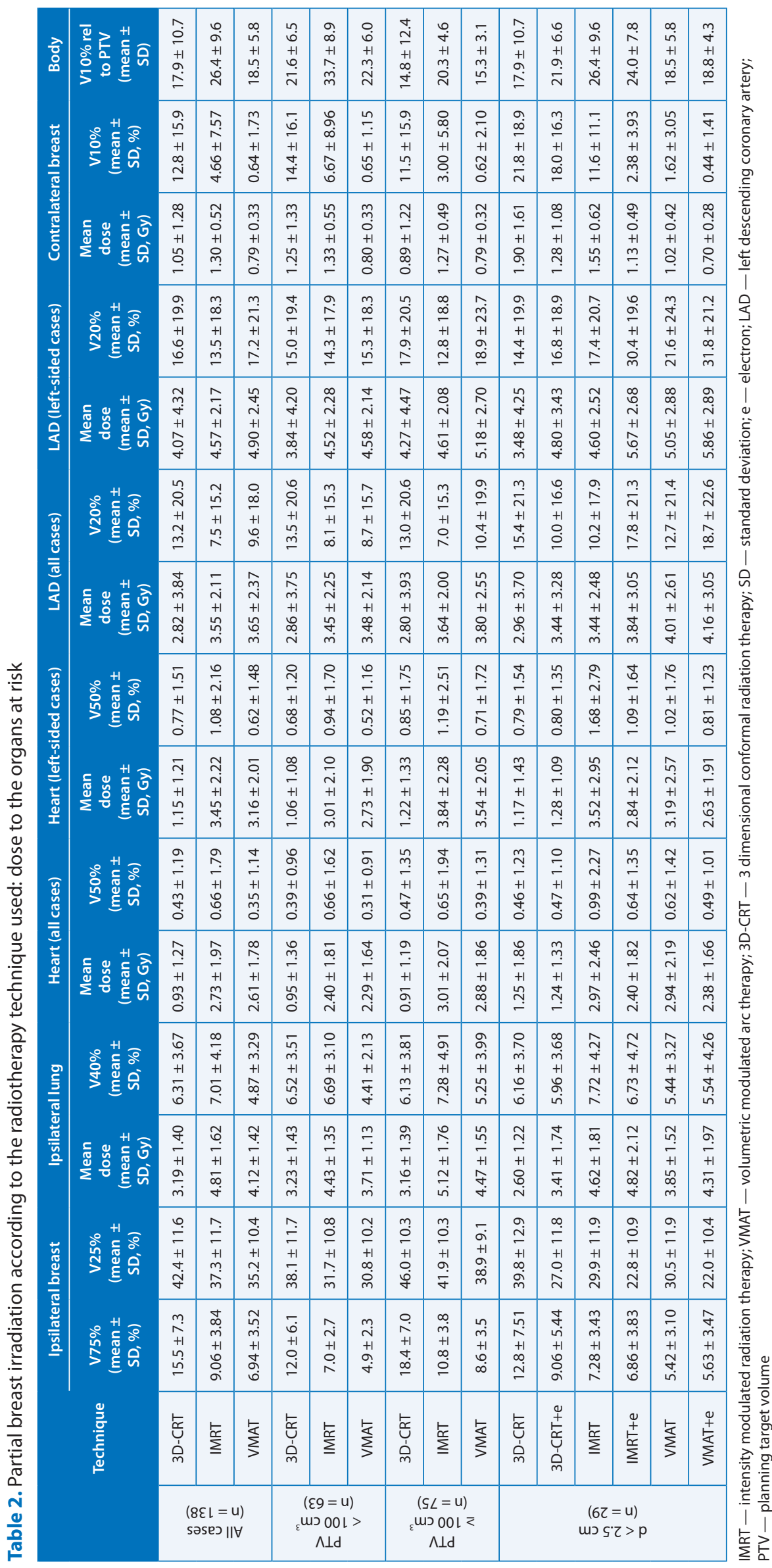


Table 3. Healthy tissue conformity $(\mathrm{H})$, merit function $(\mathrm{M})$, penalty function $(\mathrm{P})$ and plan quality index $(\mathrm{PQI})$ according to technique

\begin{tabular}{|c|c|c|c|c|c|}
\hline & Technique & $\begin{array}{c}\mathrm{H} \\
(\text { mean } \pm \mathrm{SD})\end{array}$ & $\begin{array}{c}M \\
(\text { mean } \pm S D)\end{array}$ & $\begin{array}{c}P \\
(\text { mean } \pm S D)\end{array}$ & $\begin{array}{c}\text { PQI } \\
(\text { mean } \pm S D)\end{array}$ \\
\hline \multirow{3}{*}{ All cases } & 3D-CRT & $0.598 \pm 0.067$ & $0.768 \pm 0.069$ & $0.654 \pm 0.160$ & $0.595 \pm 0.127$ \\
\hline & IMRT & $0.857 \pm 0.087$ & $0.902 \pm 0.032$ & $0.544 \pm 0.131$ & $0.497 \pm 0.126$ \\
\hline & VMAT & $0.922 \pm 0.035$ & $0.868 \pm 0.054$ & $0.571 \pm 0.128$ & $0.461 \pm 0.125$ \\
\hline \multirow{3}{*}{ PTV $<100 \mathrm{~cm}^{3}$} & 3D-CRT & $0.602 \pm 0.064$ & $0.771 \pm 0.068$ & $0.663 \pm 0.177$ & $0.588 \pm 0.137$ \\
\hline & IMRT & $0.836 \pm 0.098$ & $0.901 \pm 0.035$ & $0.591 \pm 0.120$ & $0.464 \pm 0.115$ \\
\hline & VMAT & $0.923 \pm 0.039$ & $0.865 \pm 0.062$ & $0.613 \pm 0.117$ & $0.424 \pm 0.113$ \\
\hline \multirow{3}{*}{ PTV $\geq 100 \mathrm{~cm}^{3}$} & 3D-CRT & $0.594 \pm 0.070$ & $0.765 \pm 0.070$ & $0.647 \pm 0.145$ & $0.601 \pm 0.119$ \\
\hline & IMRT & $0.876 \pm 0.072$ & $0.903 \pm 0.030$ & $0.505 \pm 0.127$ & $0.524 \pm 0.129$ \\
\hline & VMAT & $0.921 \pm 0.033$ & $0.871 \pm 0.046$ & $0.535 \pm 0.126$ & $0.492 \pm 0.128$ \\
\hline \multirow{6}{*}{$\mathrm{d}<2.5 \mathrm{~cm}$} & 3D-CRT & $0.604 \pm 0.071$ & $0.753 \pm 0.059$ & $0.651 \pm 0.223$ & $0.607 \pm 0.169$ \\
\hline & 3D-CRT+e & $0.799 \pm 0.082$ & $0.704 \pm 0.072$ & $0.673 \pm 0.155$ & $0.505 \pm 0.120$ \\
\hline & IMRT & $0.811 \pm 0.089$ & $0.882 \pm 0.040$ & $0.576 \pm 0.154$ & $0.495 \pm 0.133$ \\
\hline & IMRT+e & $0.870 \pm 0.069$ & $0.789 \pm 0.059$ & $0.611 \pm 0.134$ & $0.475 \pm 0.113$ \\
\hline & VMAT & $0.893 \pm 0.042$ & $0.824 \pm 0.065$ & $0.568 \pm 0.167$ & $0.490 \pm 0.149$ \\
\hline & VMAT+e & $0.916 \pm 0.048$ & $0.778 \pm 0.059$ & $0.611 \pm 0.136$ & $0.467 \pm 0.118$ \\
\hline
\end{tabular}

IMRT — intensity modulated radiation therapy; VMAT — volumetric modulated arc therapy; 3D-CRT — 3 dimensional conformal radiation therapy; SD — standard deviation; e - electron; PTV — planning target volume

the 3D-CRT was the favourable method (3D-CRT vs. IMRT: $\mathrm{p}=0.035,3 \mathrm{D}-\mathrm{CRT}$ s. VMAT: $\mathrm{p}=0.002$, 3D-CRT vs. IMRT/VMAT: $\mathrm{p}=0.019$ ).

The sole comparison of IMRT and VMAT techniques indicated that IMRT plans provided superior PQI values in superficial PTV cases $(\mathrm{p}<0.001)$

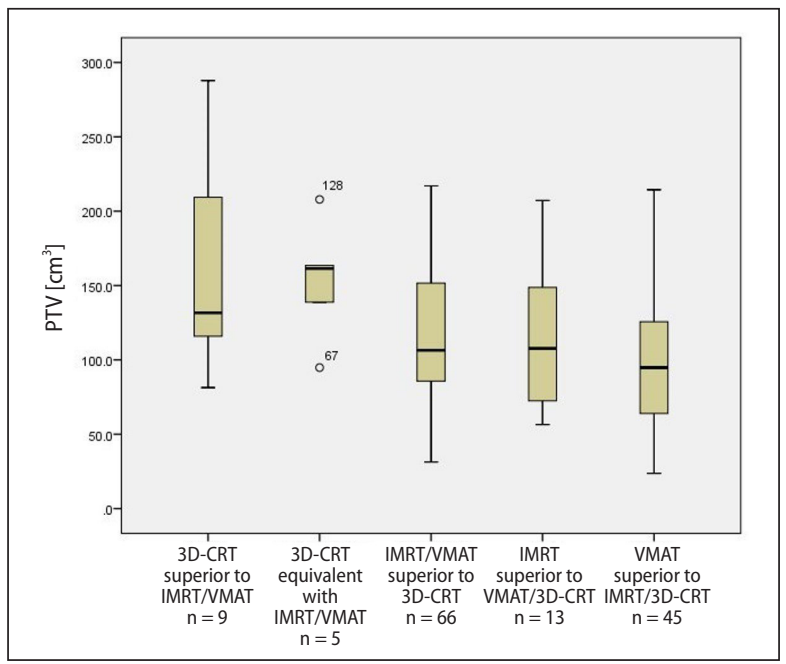

Figure 2. Comparison of $\mathrm{PQI}$ values. $\mathrm{PQI}$ values of those patients for whom 3D-CRT was the most advantageous, 3D-CRT was equivalent with IMRT or VMAT, IMRT and VMAT were equivalent but superior to 3D-CRT, IMRT was the most favourable and finally VMAT was the most favourable plan, depending on the volume of the PTV
(Fig. 3) and tumour beds situated in the upper $(\mathrm{p}<0.046)$ and medial/central $(\mathrm{p}<0.032)$ breast quadrants (Tab. 4).

In the case of superficial tumour beds $(\mathrm{d}<25$ $\mathrm{mm}, 29$ patients), the impact of an added electron beam was studied for each technique (3D-CRT, IMRT and VMAT). Two-way repeated measures

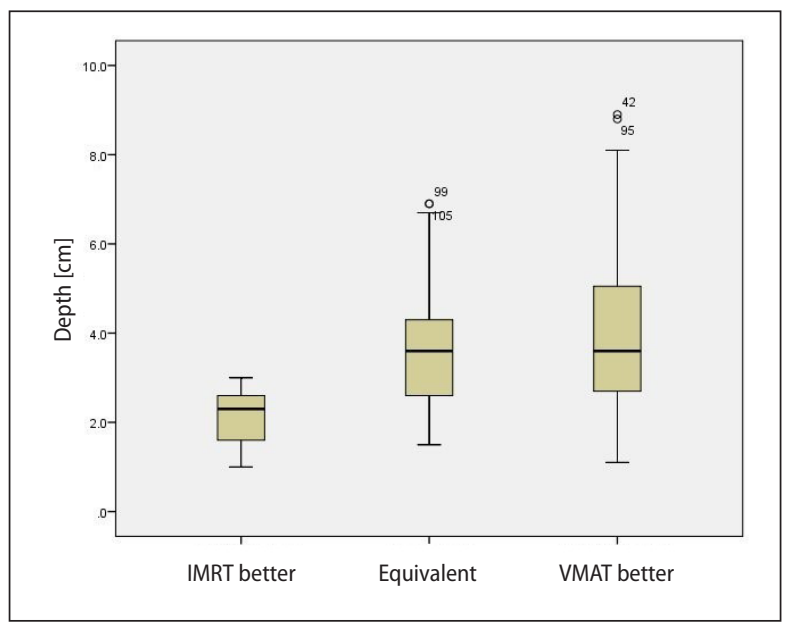

Figure 3. Comparison of intensity modulated radiation therapy (IMRT) and volumetric modulated arc therapy (VMAT) techniques. Plan quality index (PQI) was superior with IMRT in cases with superficially located target volumes than with VMAT 
Table 4. The more advantageous radiotherapy technique in relation to the location of target volume

\begin{tabular}{|c|c|c|c|c|c|c|c|c|}
\hline & \multicolumn{3}{|c|}{ Radiotherapy technique [n (\%)] } & & \multicolumn{3}{|c|}{ Radiotherapy technique [n (\%)] } \\
\hline & & IMRT better & Equiva-lent & VMAT better & & IMRT better & Equiva-lent & VMAT better \\
\hline \multirow{2}{*}{ Quadrant } & Lateral & $4(28.6 \%)$ & $44(63.8 \%)$ & $36(65.5 \%)$ & Lower & $0(0 \%)$ & $21(30.4 \%)$ & $12(21.8 \%)$ \\
\hline & Medial/central & 10 (71.4\%) & 25 (36.2\%) & 19 (34.5\%) & Upper & $14(100 \%)$ & 48 (69.6\%) & 43 (78.2\%) \\
\hline
\end{tabular}

IMRT — intensity modulated radiation therapy; VMAT — volumetric modulated arc therapy

Table 5. Mean differences of plan quality index (PQI) values regarding the effect of adding an 'en face' electron beam to photon beams using IMRT, VMAT and 3D-CRT techniques

\begin{tabular}{|l|c|c|c|c|}
\hline \multicolumn{2}{|c|}{ Mean \pm SD of PQI } & \multirow{2}{*}{ PQID } & 95\% Confidence interval for PQID & P \\
\cline { 1 - 2 } IMRT & $0.495 \pm 0.025$ & $0.000-0.039$ & 0.055 \\
\cline { 1 - 2 } IMRT + electron & $0.475 \pm 0.021$ & 0.023 & $0.002-0.045$ & \multirow{2}{*}{0.037} \\
\cline { 1 - 2 } VMAT & $0.490 \pm 0.028$ & 0.102 & $0.070-0.133$ & $<0.001$ \\
\hline VMAT + electron & $0.467 \pm 0.022$ & & \\
\hline 3D-CRT & $0.607 \pm 0.031$ & $0.505 \pm 0.022$ & & \\
\hline
\end{tabular}

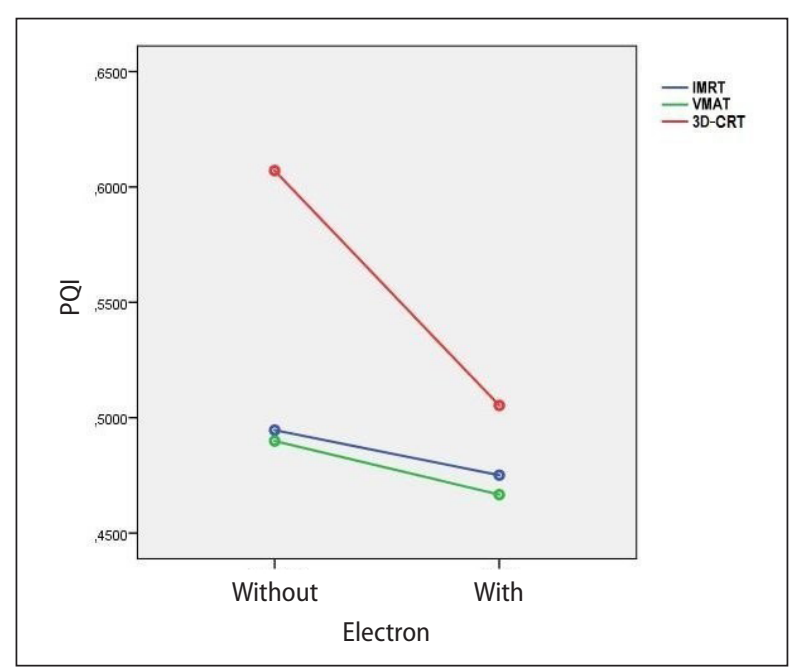

Figure 4. Effect of adding "en face" electron beam to photon beams on intensity modulated radiation therapy (IMRT), volumetric modulated arc therapy (VMAT), 3 dimensional conformal radiation therapy (3D-CRT) plans. An additional electron beam improved the plan quality index (PQI) of all the studied RT techniques, nevertheless, this was relevant for 3D-CRT plans only

ANOVA proved that the magnitude of the effect is determined by the given technique (significant interaction, $\mathrm{p}<0.001$ ). An additional electron beam improved the PQI of all the studied RT techniques; nevertheless, this was relevant (PQID > 0.05) for 3D-CRT plans only (Tab. 5, Fig. 4). In 67 patients with PQID $>0.05$, the role of the various components $(\mathrm{H}, \mathrm{M}$ and $\mathrm{P})$ of the PQI was analysed. In fact, the PQI values mostly differed according to the $\mathrm{P}$ function (risk organ exposure). This function was the strength of a few $(n=9) 3 D$-CRT-preferred cases with a relatively large PTV (mean: $159.3 \mathrm{~cm}^{3}$, range: $81.3-287.8 \mathrm{~cm}^{3}$ ) (Fig. 5).

\section{Discussion}

In selected patients with early breast cancer APBI is an attractive treatment approach and a rational alternative to conventional postoperative whole breast irradiation by shortening RT course and decreasing OAR exposure significantly $[1,3,4]$. For APBI, several external beam techniques with varying dosimetry have been investigated [8, 9, 21-25]. According to our findings, in a relevant proportion of patients 3D-CRT, IMRT or VMAT may give individually superior results, and dose homogeneity and risk organ protection could be improved with appropriate technique selection. The here described PQI method embraces homogeneity, conformity, and complex OAR exposure, and may be applied as a comprehensive tool for comparing teletherapy techniques used for APBI.

The dosimetry of IMRT and VMAT over the standard conformal technique has been analysed in several studies [12, 26-31]. The use of inverse treatment planning improved conformity and, in most cases, also the exposure of selected risk organs decreased. With IMRT, the dose to the lung and heart [27] and 


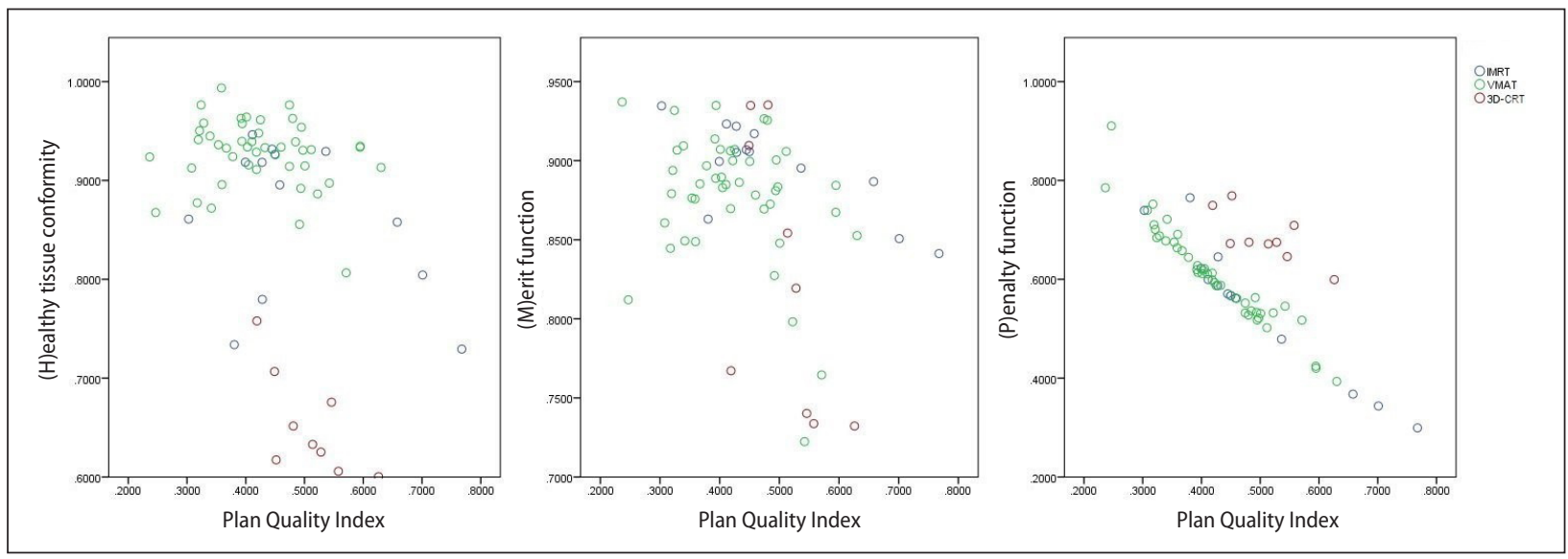

Figure 5. Representation of the effect of plan quality index (PQI) components according to the preferable plan (IMRT, VMAT, $3 \mathrm{D}-\mathrm{CRT}$ ). $\mathrm{PQI}$ values mostly differed according to the $\mathrm{P}$ function (risk organ exposure)

the ipsilateral breast $[26,27]$ was reduced as compared to 3D-CRT. According to Rusthoven et al. [27], ipsilateral breast exposure was especially lower with IMRT as compared to 3D-CRT in patients with high PTV/breast volume ratios and smaller breast sizes. Using the complex PQI formula, we identified 9/138 cases with relatively large PTVs for whom 3D-CRT was the most preferable technique. VMAT provided lower lung and heart doses than 3D-CRT [31]. Qiu et al. [29] reported the dosimetric analysis of $163 \mathrm{D}$ CRT vs. IMRT vs. VMAT plans. As compared to 3DCRT or IMRT, the use of VMAT resulted in significantly lower ipsilateral breast doses $\left(\mathrm{V}_{5 \mathrm{~Gy}}, \mathrm{~V}_{\text {10Gy }}\right)$. The lung doses were more favourable with the inverse treatment techniques while the IMRT technique yielded the best low-dose distribution in the ipsilateral lung [29]. There was no significant difference in heart exposure among the three methods.

Stelczer et al. [30] compared the step and shoot and sliding window IMRT and VMAT methods to the 3D-CRT technique based on various dosimetric parameters and used the original PQI approach [18] in 40 low-risk breast cancer cases. While dose homogeneity was superior using sliding window IMRT, in accordance with our results, ipsilateral breast exposure was significantly lower with VMAT, and the protection of the lung and heart was the best with 3D-CRT [30]. $\mathrm{V}_{50 \%}$ of the ipsilateral breast was the lowest in VMAT plans (29.4\%) as compared to 3D-CRT (44.1\%) and sliding window IMRT (35.6\%) [30]. Based on these findings, the authors recommend the sliding window IMRT technique for APBI.
In most reports [12, 26-31], unlike to our tangential field 3D-CRT technique a 5/6-field non-coplanar 3D-CRT method was applied. Only Kirby et al. [32] used 2 tangential fields in their early dosimetry analysis. In order to find out how heart doses vary if 5-6 fields are used instead of 2 tangential fields, we generated such 3D-CRT plans in 20 left-sided cases with tumour beds in the lower quadrants. The 5/6-field method provided the most benefit in lowering high-dose healthy breast tissue volumes (ipsilateral and contralateral) and improved $\mathrm{CN}$, but both low-dose body volumes and heart and LAD doses were increased as compared to that with the tangential 2-field technique (data not shown). We conclude that although the 5/6-field non-coplanar 3D-CRT technique may substitute the advanced radiotherapy techniques (nevertheless, its drawbacks may be longer treatment time and risk of geographical miss), the 2-field tangential 3D-CRT technique may serve as a rescue technique in difficult cases if heart/LAD avoidance is a priority. In centres where all teletherapy techniques (including IMRT/VMAT) are available, in selected cases the tangential-field 3DCRT may provide a solution to prevent radiogenic heart damage. We think that the non-coplanar 5-field 3D-CRT technique should be preferred in those centres where the advanced techniques are not available. Most patients in our study needed (and received if available) the IMRT/VMAT technique, but still, in a few cases, tangential field 3DCRT was the solution with respect to heart doses. The addition of an electron beam to photons im- 
proved conformality but deteriorated homogeneity. According to five reports [11-14, 33] about the application of the mixed beam technique, the addition of electrons to photon fields decreased the ipsilateral breast dose while the heart and lung doses were different according to the study and obviously the PTV location [14]. Evidently, the use of an additional electron beam is feasible only in the case of superficial tumour beds [10]. According to our results, the use of 3D-CRT and a supplementary electron field was beneficial in patients with a tumour bed situated $<25 \mathrm{~mm}$ from the body surface. We think that this technique could be suggested if, due to restricted resources and access to advanced technology, 3D-CRT was implemented.

In selected cases, APBI shows comparable efficacy and less side effects together with better cosmetic results and improved patient acceptance as compared to whole breast RT [34, 35]. Several prospective phase II and III trials employing $3 \mathrm{D}$ CRT reported a favourable early and late toxicity profile, good or excellent cosmesis and quality of life comparable with that with whole breast RT [22, 35-37]. In general, similarly good outcome was published with IMRT $[24,25]$. However, some studies implementing IMRT or 3D-CRT for APBI, reported progressive breast fibrosis and unfavourable cosmesis [23, 26, 38-42]. According to the recent RAPID study, more cases with fibrosis and progressively worsening cosmetic results were identified after APBI with 3D-CRT or IMRT as compared to those after whole breast irradiation [41]. In these trials doses comparable to other external beam APBI studies were delivered, however, in an accelerated way (twice-daily dosing). In the UK IMPORT LOW [35] and the Florence University [43] studies applying 3D-CRT or IMRT not with accelerated fractionation cosmesis was superior in the APBI arms $[35,43]$. Deteriorated cosmesis after APBI with 3D-CRT or IMRT could also be the consequence of larger PTVs and the irradiation of higher ipsilateral breast tissue volume. The relationship between large irradiated volumes and impaired cosmetic outcome due to fibrosis has been reported [44]. Based on our data, for enhanced ipsilateral breast protection we suggest the implementation of VMAT or, in the case of 3D-CRT, the additional use of electron fields.

Our results suggest that, while all three methods provide similar PTV-coverage and homoge- neity, a significant difference may occur in risk organ doses in half of the patients: heart and LAD and ipsilateral breast radiation exposure may vary according to the radiation technique and could be individually selected. For the evaluation of different techniques, various measures have been reported. Most of the studies compared various dose-volume parameters including risk organ exposure, maximum doses, coverage, conformation number, conformity, and homogeneity index or even the PQI [18]. All of them reflect a different perspective; therefore, comparing them alone is challenging. Hence, we endeavoured to use a comprehensive tool, considering homogeneity, conformity, and OAR exposure concurrently. Since our results indicate that the studied teletherapy methods are similar regarding homogeneity and conformity but may cause individually different OAR exposures, we propose to select a specific technique by focusing on maximum OAR protection. The PQI was developed for plan comparison, but not for routine practice. Based on our experience, we rather recommend specifying APBI OAR dose constraints for the heart, LAD, lung, ipsilateral and contralateral breast. IMRT/VMAT fits most cases, however, in rare cases another more appropriate technique may be needed. In large PTVs the tangential-field 3D-CRT (in inner-quadrant tumour bed cases with small PTVs), the mixed-field technique may give superior results. The probability and severity of various radiation risks must be weighted individually. In view of individualized radiotherapy especially in low risk-like APBI cases, the appropriate radiotherapy technique should be chosen based on the individual risk profile of the patient.

\section{Conclusions}

We consider PQI an appropriate approach for the evaluation of teletherapy APBI plans. In general, IMRT and particularly VMAT technique provides preferable $\mathrm{PQI}$ values as compared to that with $3 \mathrm{D}$ CRT. Applying 3D-CRT may be advantageous in patients with large tumour beds. In case of superficial PTVs, a supplementary electron field improves the PQI value of 3D-CRT plans significantly. IMRT plans may be superior if the target volume is situated in the superior or inner quadrant of the breast. PQI is mainly determined by OAR exposure. 


\section{Acknowledgements}

The authors greatly appreciate all the support and work of high standard provided by physician, physicist and technician colleagues of the Department of Oncotherapy, University of Szeged.

\section{Conflicts of interest}

None declared.

\section{Funding}

This publication was prepared without any external source of funding.

\section{References}

1. Veronesi U, Marubini E, Mariani L, et al. Radiotherapy after breast-conserving surgery in small breast carcinoma: long-term results of a randomized trial. Ann Oncol. 2001; 12(7): 997-1003, doi: 10.1023/a:1011136326943, indexed in Pubmed: 11521809.

2. Vicini FA, Arthur DW. Breast brachytherapy: North American experience. Semin Radiat Oncol. 2005; 15(2): 108-115, doi: 10.1016/j.semradonc.2004.10.005, indexed in Pubmed: 15809936.

3. Polgár C, Strnad V, Major T. Brachytherapy for partial breast irradiation: the European experience. Semin Radiat Oncol. 2005; 15(2): 116-122, doi: 10.1016/j.semradonc.2004.10.004, indexed in Pubmed: 15809937.

4. Polgár C, Van Limbergen E, Pötter R, et al. GEC-ESTRO breast cancer working group. Patient selection for accelerated partial-breast irradiation (APBI) after breast-conserving surgery: recommendations of the Groupe Européen de Curiethérapie-European Society for Therapeutic Radiology and Oncology (GEC-ESTRO) breast cancer working group based on clinical evidence (2009). Radiother Oncol. 2010; 94(3): 264-273, doi: 10.1016/j.radonc.2010.01.014, indexed in Pubmed: 20181402.

5. Smith BD, Arthur DW, BuchholzTA, et al. Accelerated partial breast irradiation consensus statement from the American Society for Radiation Oncology (ASTRO). Int J Radiat Oncol Biol Phys. 2009; 74(4): 987-1001, doi: 10.1016/j. ijrobp.2009.02.031, indexed in Pubmed: 19545784.

6. Shah C, Wobb J, Manyam B, et al. Accelerated partial breast irradiation utilizing brachytherapy: patient selection and workflow. J Contemp Brachytherapy. 2016; 8(1): 90-94, doi: $10.5114 / \mathrm{jcb} .2016 .58083$, indexed in Pubmed: 26985202.

7. Correa C, Harris EE, Leonardi MC, et al. Accelerated Partial Breast Irradiation: Executive summary for the update of an ASTRO Evidence-Based Consensus Statement. Pract Radiat Oncol. 2017; 7(2): 73-79, doi: 10.1016/j. prro.2016.09.007, indexed in Pubmed: 27866865.

8. Njeh CF, Saunders MW, Langton CM, et al. Accelerated Partial Breast Irradiation (APBI): A review of available techniques. Radiat Oncol. 2010; 5(1): 90-20, doi: 10.1186/1748717X-5-90, indexed in Pubmed: 20920346.

9. Formenti SC. External-beam partial-breast irradiation. Semin Radiat Oncol. 2005; 15(2): 92-99, doi: 10.1016/j. semradonc.2004.10.008, indexed in Pubmed: 15809934.
10. Fekete G, Újhidy D, Együd Z, et al. Partial breast radiotherapy with simple teletherapy techniques. Med Dosim. 2015; 40(4): 290-295, doi: 10.1016/j.meddos.2015.03.006, indexed in Pubmed: 25899875.

11. Kozak KR, Doppke KP, Katz A, et al. Dosimetric comparison of two different three-dimensional conformal external beam accelerated partial breast irradiation techniques. Int J Radiat Oncol Biol Phys. 2006; 65(2): 340-346, doi: 10.1016/j.ijrobp.2005.12.053, indexed in Pubmed: 16690427.

12. El Nemr M, Heymann S, Verstraet R, et al. Mixed modality treatment planning of accelerated partial breast irradiation: to improve complex dosimetry cases. Radiat Oncol. 2011; 6: 154, doi: 10.1186/1748-717X-6-154, indexed in Pubmed: 22074547.

13. Recht A, Ancukiewicz M, Alm El-Din MA, et al. Lung dose-volume parameters and the risk of pneumonitis for patients treated with accelerated partial-breast irradiation using three-dimensional conformal radiotherapy. J Clin Oncol. 2009; 27(24): 3887-3893, doi: 10.1200/ JCO.2008.20.0121, indexed in Pubmed: 19620489.

14. Mydin AR, Gaffney H, Bergman A, et al. Does a three-field electron/minitangent photon technique offer dosimetric advantages to a multifield, photon-only technique for accelerated partial breast irradiation? Am J Clin Oncol. 2010; 33(4): 336-340, doi: 10.1097/COC.0b013e3181b0c370, indexed in Pubmed: 19884806.

15. van't Riet A, Mak AC, Moerland MA, et al. A conformation number to quantify the degree of conformality in brachytherapy and external beam irradiation: application to the prostate. Int J Radiat Oncol Biol Phys. 1997; 37(3): 731-736, doi: 10.1016/s0360-3016(96)00601-3, indexed in Pubmed: 9112473.

16. Shaw E, Kline R, Gillin M, et al. Radiation Therapy Oncology Group: radiosurgery quality assurance guidelines. Int J Radiat Oncol Biol Phys. 1993; 27(5): 1231-1239, doi: 10.1016/0360-3016(93)90548-a, indexed in Pubmed: 8262852.

17. Lomax NJ, Scheib SG. Quantifying the degree of conformity in radiosurgery treatment planning. Int J Radiat Oncol Biol Phys. 2003; 55(5): 1409-1419, doi: 10.1016/ s0360-3016(02)04599-6, indexed in Pubmed: 12654454.

18. Leung LH, Kan MW, Cheng AC, et al. A new dose-volumebased Plan Quality Index for IMRT plan comparison. Radiother Oncol. 2007; 85(3): 407-417, doi: 10.1016/j. radonc.2007.10.018, indexed in Pubmed: 18023487.

19. Duane F, Aznar MC, Bartlett F, et al. A cardiac contouring atlas for radiotherapy. Radiother Oncol. 2017; 122(3): 416-422, doi: 10.1016/j.radonc.2017.01.008, indexed in Pubmed: 28233564.

20. Varga Z, Cserháti A, Rárosi F, et al. Individualized positioning for maximum heart protection during breast irradiation. Acta Oncol. 2014; 53(1): 58-64, doi: 10.3109/028418 6X.2013.781674, indexed in Pubmed: 23544358.

21. Chen PY, Wallace M, Mitchell $C$, et al. Four-year efficacy, cosmesis, and toxicity using three-dimensional conformal external beam radiation therapy to deliver accelerated partial breast irradiation. Int J Radiat Oncol Biol Phys. 2010; 76(4): 991-997, doi: 10.1016/j.ijrobp.2009.03.012, indexed in Pubmed: 19515514.

22. Shah C, Wilkinson JB, Lanni T, et al. Five-year outcomes and toxicities using 3-dimensional conformal external beam radiation therapy to deliver accelerated par- 
tial breast irradiation. Clin Breast Cancer. 2013; 13(3): 206-211, doi: 10.1016/j.clbc.2012.09.020, indexed in Pubmed: 23103365.

23. Liss AL, Ben-David MA, Jagsi R, et al. Decline of cosmetic outcomes following accelerated partial breast irradiation using intensity modulated radiation therapy: results of a single-institution prospective clinical trial. Int J Radiat Oncol Biol Phys. 2014; 89(1): 96-102, doi: 10.1016/j. ijrobp.2014.01.005, indexed in Pubmed: 24613813.

24. Lewin AA, Derhagopian R, Saigal K, et al. Accelerated partial breast irradiation is safe and effective using intensity-modulated radiation therapy in selected early-stage breast cancer. Int J Radiat Oncol Biol Phys. 2012; 82(5): 2104-2110, doi: 10.1016/j.jirobp.2011.02.024, indexed in Pubmed: 21640490.

25. Lei RY, Leonard CE, Howell KT, et al. Four-year clinical update from a prospective trial of accelerated partial breast intensity-modulated radiotherapy (APBIMRT). Breast Cancer Res Treat. 2013; 140(1): 119-133, doi: 10.1007/ s10549-013-2623-x, indexed in Pubmed: 23824363.

26. Moon SHo, Shin KH, Kim TH, et al. Dosimetric comparison of four different external beam partial breast irradiation techniques: three-dimensional conformal radiotherapy, intensity-modulated radiotherapy, helical tomotherapy, and proton beam therapy. Radiother Oncol. 2009; 90(1): 66-73, doi: 10.1016/j.radonc.2008.09.027, indexed in Pubmed: 18992950.

27. Rusthoven KE, Carter DL, Howell K, et al. Accelerated partial-breast intensity-modulated radiotherapy results in improved dose distribution when compared with threedimensional treatment-planning techniques. Int J Radiat Oncol Biol Phys. 2008; 70(1): 296-302, doi: 10.1016/j. ijrobp.2007.08.047, indexed in Pubmed: 18086392.

28. Qiu JJ, Chang Z, Wu QJ, et al. Impact of volumetric modulated arc therapy technique on treatment with partial breast irradiation. Int J Radiat Oncol Biol Phys. 2010; 78(1): 288-296, doi: 10.1016/j.ijrobp.2009.10.036, indexed in Pubmed: 20444558.

29. Qiu JJ, Chang Z, Horton JK, et al. Dosimetric comparison of 3D conformal, IMRT, and V-MAT techniques for accelerated partial-breast irradiation (APBI). Med Dosim. 2014; 39(2): 152-158, doi: 10.1016/j.meddos.2013.12.001, indexed in Pubmed: 24480375.

30. Stelczer G, MajorT, Mészáros N, et al. External beam accelerated partial breast irradiation: dosimetric assessment of conformal and three different intensity modulated techniques. Radiol Oncol. 2019; 53(1): 123-130, doi: 10.2478/ raon-2019-0001, indexed in Pubmed: 30661060.

31. Essers M, Osman SOS, Hol S, et al. Accelerated partial breast irradiation (APBI): are breath-hold and volumetric radiation therapy techniques useful? Acta Oncol. 2014; 53(6): 788-794, doi: 10.3109/0284186X.2014.887226, indexed in Pubmed: 24689645.

32. Kirby AM, Evans PM, Donovan EM, et al. Prone versus supine positioning for whole and partial-breast radiotherapy: a comparison of non-target tissue dosimetry. Radiother Oncol. 2010; 96(2): 178-184, doi: 10.1016/j. radonc.2010.05.014, indexed in Pubmed: 20561695.

33. Palma BA, Sánchez AU, Salguero FJ, et al. Combined modulated electron and photon beams planned by a Monte-Carlo-based optimization procedure for accelerated partial breast irradiation. Phys Med Biol. 2012; 57(5):
1191-1202, doi: 10.1088/0031-9155/57/5/1191, indexed in Pubmed: 22330241.

34. Strnad V, Ott OJ, Hildebrandt G, et al. Groupe Européen de Curiethérapie of European Society for Radiotherapy and Oncology (GEC-ESTRO). 5-year results of accelerated partial breast irradiation using sole interstitial multicatheter brachytherapy versus whole-breast irradiation with boost after breast-conserving surgery for low-risk invasive and in-situ carcinoma of the female breast: a randomised, phase 3, non-inferiority trial. Lancet. 2016; 387(10015): 229-238, doi: 10.1016/S0140-6736(15)00471-7, indexed in Pubmed: 26494415.

35. Coles CE, Griffin CL, Kirby AM, et al. IMPORTTrialists. Partialbreast radiotherapy after breast conservation surgery for patients with early breast cancer (UK IMPORT LOW trial): 5 -year results from a multicentre, randomised, controlled, phase 3, non-inferiority trial. Lancet. 2017; 390(10099): 1048-1060, doi: 10.1016/S0140-6736(17)31145-5, indexed in Pubmed: 28779963.

36. Rodríguez N, Sanz X, Dengra J, et al. Five-year outcomes, cosmesis, and toxicity with 3-dimensional conformal external beam radiation therapy to deliver accelerated partial breast irradiation. Int J Radiat Oncol Biol Phys. 2013; 87(5): 1051-1057, doi: 10.1016/j.jijrobp.2013.08.046, indexed in Pubmed: 24161420.

37. Chafe S, Moughan J, McCormick B, et al. Late toxicity and patient self-assessment of breast appearance/satisfaction on RTOG 0319: a phase 2 trial of 3-dimensional conformal radiation therapy-accelerated partial breast irradiation following lumpectomy for stages I and II breast cancer. Int J Radiat Oncol Biol Phys. 2013; 86(5): 854-859, doi: 10.1016/j.ijrobp.2013.04.005, indexed in Pubmed: 23726000.

38. Jagsi R, Ben-David MA, Moran JM, et al. Unacceptable cosmesis in a protocol investigating intensity-modulated radiotherapy with active breathing control for accelerated partial-breast irradiation. Int J Radiat Oncol Biol Phys. 2010; 76(1): 71-78, doi: 10.1016/j.ijrobp.2009.01.041, indexed in Pubmed: 19409733.

39. Hepel JT, Tokita M, MacAusland SG, et al. Toxicity of threedimensional conformal radiotherapy for accelerated partial breast irradiation. Int J Radiat Oncol Biol Phys. 2009; 75(5): 1290-1296, doi: 10.1016/j.ijrobp.2009.01.009, indexed in Pubmed: 19395195.

40. Olivotto IA, Whelan TJ, Parpia S, et al. Interim cosmetic and toxicity results from RAPID: a randomized trial of accelerated partial breast irradiation using three-dimensional conformal external beam radiation therapy. J Clin Oncol. 2013;31(32): 4038-4045, doi: 10.1200/JCO.2013.50.5511, indexed in Pubmed: 23835717.

41. Whelan T, Julian J, Levine M, et al. RAPID: A randomized trial of accelerated partial breast irradiation using 3-dimensional conformal radiotherapy (3DCRT). Cancer Res. 2019; 79(4 Suppl): GS4-03.

42. Vicini FA, Cecchini RS, White JR, et al. Long-term primary results of accelerated partial breast irradiation after breast-conserving surgery for early-stage breast cancer: a randomised, phase 3 , equivalence trial. Lancet. 2019; 394(10215): 2155-2164, doi: 10.1016/S01406736(19)32514-0, indexed in Pubmed: 31813636.

43. Meattini I, Marrazzo L, Saieva C, et al. Accelerated PartialBreast Irradiation Compared With Whole-Breast Irra- 
diation for Early Breast Cancer: Long-Term Results of the Randomized Phase III APBI-IMRT-Florence Trial. J Clin Oncol. 2020; 38(35): 4175-4183, doi: 10.1200/JCO.20.00650, indexed in Pubmed: 32840419.
44. Borger JH, Kemperman H, Smitt HS, et al. Dose and volume effects on fibrosis after breast conservation therapy. Int J Radiat Oncol Biol Phys. 1994; 30(5): 1073-1081, doi: 10.1016/03603016(94)90312-3, indexed in Pubmed: 7961014. 